Letter to the Editor

S Kiani-Alikhan, E Walker, A Hickey, M Buckland, C Scott Corresponding Author's given name initial(s). S

Kiani-Alikhan

\title{
Measurement of C1-Inhibitor function alone is sufficient for diagnosis of hereditary angioedema
}

https://doi.org/xxxxx/xxxxxxxxxx

Received Month Day, Year; accepted Month Day, Year; previously published online Month Day, Year

Keywords: HAE, C1-Inhibitor, Angioedema, C1-Esterase Inhibitor

To the Editor

Hereditary angioedema (HAE) types 1 and 2 is anare autosomal dominant disorders due to reduced levels or dysfunctional $\mathrm{C} 1$-Inhibitor $(\mathrm{C} 1-\mathrm{INH})$. This results in dysregulation of the contact kinin system and over production of bradykinin, which causes extravasation of fluid and recurrent debilitating and life-threatening subcutaneous and submucosal swellings. The WAO/EAACI (2018) consensus guidelines 1on HAE state that "all patients suspected to have HAE-1/2 [should be] assessed for blood levels of C1-INH function, C1-INH protein, and Complement4 (C4). If the levels of any of these analytes are abnormally low, the tests should be repeated to confirm the diagnosis of HAE-1/2." The guidelines do not comment on other testing paradigms and in practice $\mathrm{C} 4$ with or without $\mathrm{C} 1$-Inhibitor levels are tested in most immunology laboratories. A low $\mathrm{C} 4$ can be observed during an attack but in many patients it remains low in between attacks and hence, it can be used for screening but it could miss a number of patients. C1-INH levels can be normal in patients with HAE type 2. C1-INH function is low in both HAE 1 and 2 patients. C1-INH levels could can be accurately measured by radial immunodiffusion (RID). It uses small amounts of serum and has high analytic sensitivity but apart from not being suitable for HAE type2, it is time consuming. It needs set up time and reading time 72 hours later. The newer methods are mostly used on automated analysers that require larger serum volumes and so problematic in testing small children. The cost of $\mathrm{C} 1-\mathrm{INH}$ antigenic level and $\underline{\mathrm{fF}}$-unction are very similar, but $\mathrm{C} 4$ is much cheaper than both.

We analysed the requests to our laboratory for C1-INH level and function over a 6 months period to April 2019. A total of 441 requests were sent to the lab, 368 of which were normal.
Of the 73 abnormal results that could be diagnosed with HAE, all $(100 \%)$ had abnormal C1-INH function but $8(10.9 \%)$ had normal C1-INH levels thus potentially missing a diagnosis of $\mathrm{HAE}$ if antigenic level only were tested. Therefore the sensitivity of C1-INH function was $100 \%$ compared to $89.1 \%$ for C1-INH levels.

Table 1. Correlation of the measurements of $\mathrm{C} 1-\mathrm{INH}$ level and Function in 441 consecutive patients.

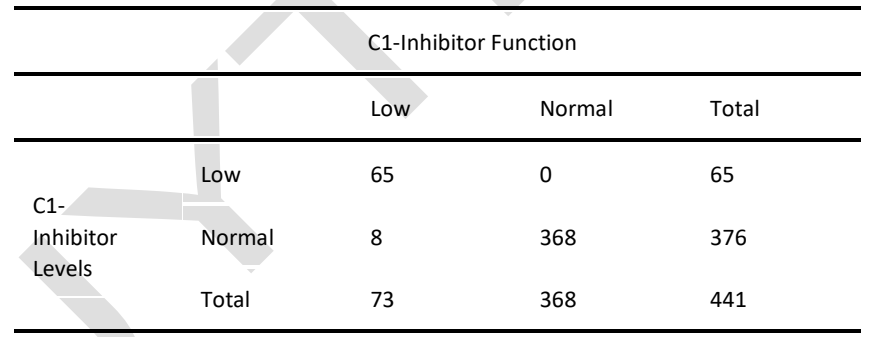

In the footnote, arefer to information within the Table with superscript lowercase letters; band do not use special characters or numbers; 'separate abbreviations by comma and semicolon. Abb1, Abbreviation1; Abb2, Abbreviation2.

The paradigm for testing in our laboratory has changed to initial measurement of $\mathrm{C} 1$-INH function followed by measuring C1-INH and C4 levels only if the function is abnormal. This change has resulted in a faster turn-around time and significant savings. Considering that HAE is a rare disease (approx. 1:25000) and majority of patients investigated do not have this condition, our data shows that this change of strategy reduces costs significantly without affecting the clinical sensitivity.

\section{References}

1. The international WAO/EAACl guideline for the management of hereditary angioedema - the 2017 revision and update. World Allergy Organization Journal201811:5

2. Karim, Y., Griffiths, H. and Deacock, S., 2004. Normal complement C4 values do not exclude hereditary angioedema. Journal of Clinical Pathology, 57(2) pp.213-214.

\footnotetext{
${ }^{\star}$ Corresponding Author(s): S Kiani-Alikhan, Immunology department Barts Health NHS Trust, London, United Kingdom, E-mail:skiani@nhs.net E Walker, A Hickey, M Buckland, C Scott: Immunology department, Barts Health NHS Trust, London, United Kingdom
} 